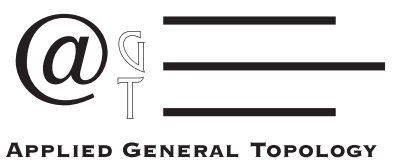

\title{
A class of spaces containing all generalized absolutely closed (almost compact) spaces
}

\author{
J. K. KoHLI AND A. K. DAS
}

\begin{abstract}
The class of $\theta$-compact spaces is introduced which properly contains the class of almost compact (generalized absolutely closed) spaces and is strictly contained in the class of quasicompact spaces. In the realm of almost regular spaces, the class of $\theta$-compact spaces coincides with the class of nearly compact spaces. Moreover, an almost regular $\theta$-compact space is mildly normal $(=\kappa$-normal $)$. A $\theta$-closed, $\theta$-embedded subset of a $\theta$-compact space is $\theta$-compact and the product of two $\theta$-compact space is $\theta$-compact if one of them is compact. A (strongly) $\theta$-continuous image of a $\theta$-compact space is $\theta$-compact (compact). A space is compact if and only if it is $\theta$-compact and $\theta$-point paracompact.
\end{abstract}

2000 AMS Classification: Primary: 54D10, 54D20, 54D30, Secondary: $54 \mathrm{C} 08,54 \mathrm{C} 10$.

Keywords: $\theta$-compact space, almost compact (generalized absolutely closed) space, nearly compact space, quasicompact space, $\theta$-point paracompact space, $\theta$-closed $(\theta$-open $)$ set, $\theta$-limit point, almost regular space, mildly normal $(\kappa$ normal) space, almost normal space, (strongly) $\theta$-continuous function, $\theta$-map, $\theta$-closed function, $\theta$-limit point.

\section{Introduction AND Preliminaries}

Compactness plays a prominent role in topology, analysis and many other branches of mathematics. Several weak variants of compactness occur in the literature which capture partial features of compactness and are thus useful in the topological/ analytical situations where the full force of compactness is not required (see [25] [26]). The variants of compactness with which we shall be dealing in this paper include almost compactness (= generalized closedness) (see [18], [17], [8], [15]); near compactness [21]; and quasicompactness (see [8], [1] and [25]). 
In Section 2, we introduce the class of $\theta$-compact spaces and establish their place in the hierarchy of compactness and certain of its variants. The class of $\theta$-compact spaces lies strictly between the class of almost compact spaces [3] and quasicompact spaces. In the realm of almost regular spaces the notions of $\theta$-compactness and near compactness are equivalent. Interrelations between $\theta$-compactness and certain weak variants of normality are investigated. It is shown that an almost regular $\theta$-compact space is mildly normal and that a Hausdorff almost regular $\theta$-compact space is almost normal. Presevation under mappings and products of $\theta$-compact spaces are considered in Section 4 . It is shown that a (strongly) $\theta$-continuous image of a $\theta$-compact space is $\theta$-compact (compact). In Section 5, we discuss characterizations of $\theta$-compact spaces and conclude with a factorization theorem that a space is compact if and only if it is both a $\theta$-compact space and a $\theta$-point paracompact space.

Let $X$ be a topological space and let $A \subset X$. Throughout the present paper the closure of a set $A$ will be denoted by $\bar{A}$ and the interior by int $A$. A point $x \in X$ is called a $\theta$-limit point [28] of $A$ if every closed neighbourhood of $x$ intersects $A$. Let $c_{\theta} A$ denotes the set of all $\theta$-limit point of $A$. The set $A$ is called $\theta$-closed if $A=\operatorname{cl}_{\theta} A$. The complement of a $\theta$-closed set will be referred to as a $\theta$-open set. It is easily verified that in a topological space every cozero set is $\theta$-open. A set $U \subset X$ is said to be regularly open [14] if $U=i n \bar{t}$. The complement of a regularly open set is called regularly closed. A space $X$ is said to be almost regular [19] if every regularly closed set and a point out side it are contained in disjoint open sets. A space $\mathrm{X}$ is said to be mildly normal [22] ( or $\kappa$-normal [24]) if every pair of disjoint regularly closed sets are contained in disjoint open sets and a space is called almost normal [20] if every pair of disjoint closed sets one of which is regularly closed are contained in disjoint open sets. A space $X$ is said to be nearly compact [21] if every open covering of $X$ admits a finite subcollection the interiors of the closures of whose members cover $X$. A space $X$ is said to be almost compact [3] if every open covering of $X$ has a finite subcollection the closures of whose members covers $X$. Almost compact spaces have been referred to as $\mathrm{H}(\mathrm{i})$ spaces by Scarborough and Stone [18] and are called generalized absolutely closed spaces by Liu [15], while Porter and Thomas [17] call them quasi-H-closed spaces. A Hausdorff almost compact space is called an $\boldsymbol{H}$-closed space. H-closed spaces have many properties similar to that of compact Hausdorff spaces. A space $X$ is said to be quasicompact [8] if every covering of $\mathrm{X}$ by cozero sets admits a finite subcollection which covers $X$. Functionally Hausdorff, quasicompact spaces are precisely the spaces in which Stone-Weierstrass theorem holds ([25], $[26])$.

Lemma 1.1 ([11, 13]). A subset $A$ of a topological space $X$ is $\theta$-open if and only if for each $x \in A$, there is an open set $U$ such that $x \in U \subset \bar{U} \subset A$.

Lemma 1.2. ([6, 2.4]). A space $X$ is regular if and only if every closed set in $X$ is $\theta$-closed. 


\section{2. $\theta$-COMPACT SPACES}

Definition 2.1. A space $X$ is said to be $\theta$-compact if every open covering of $X$ by $\theta$-open sets has a finite subcollection that covers $X$. A subset $A$ of $X$ is said to be $\theta$-compact if it is $\theta$-compact with respect to the topology it inherits as a subspace of $X$. A subset $A$ of $X$ is said to be a $\theta$-set in $X$ if every covering of $A$ by $\theta$-open sets in $X$ has a finite subcollection that covers $A$.

Remark 2.2. A $\theta$-set in a topological space need not be $\theta$-compact. For let $X=[0,1]$ with every point having usual Euclidean neighbourhood except 0 . A basic neighbourhood of 0 is of the form $U-K$, where $U$ is an Euclidean neighbourhood of 0 and $K=\{1 / n: n \in \mathrm{N}\}$. Let $A=\{0\} \cup K$. Then $A$ is a $\theta$-set in $X$ which is not $\theta$-compact.

The following implications are immediate from the definitions.

compact $\Rightarrow$ nearly compact $\Rightarrow$ almost compact $\Rightarrow \theta$-compact $\Rightarrow$ quasicompact.

However, none of the above implications is reversible.

Example 2.3. A $\theta$-compact space which is not almost compact. Let $X=\mathrm{N}$, the set of positive integers. Define a topology on $X$ by taking every odd integer to be open and a set $U$ is open if for every even integer $p \in U$, the predecessor and the successor of $p$ are also in $U$. Now the collection $\mathcal{U}=$ $\{\{2 k-1,2 k, 2 k+1\}: k \in \mathrm{N}\}$ is an open covering of $X$ which does not possess a finite subcollection whose closures covers $X$. Thus $X$ is not almost compact. However, it is $\theta$-compact.

Remark 2.4. Hewitt's example [9] of a $T_{1}$-regular space on which every continuous real-valued function is constant is a quasicompact space which is not $\theta$-compact. Singal and Mathur [21] gave an example of a nearly compact space which is not compact and an example of an almost compact space which is not nearly compact.

The following characterization of almost regular spaces besides being useful in the sequel has been extensively used in [12].

Theorem 2.5. A space $X$ is almost regular if and only if for every open set $U$ in $X$, int $\bar{U}$ is $\theta$-open.

Proof. Suppose that $U$ is an open set in $X$. If $i n t \bar{U}=X$, we are through. In case $i n t \bar{U} \neq X$ and $x \in \operatorname{int} \bar{U}$, then $x$ is not in the regularly closed set $X-i n t \bar{U}$. By almost regularity of $X$, there exist disjoint open sets $V$ and $W$ containing $x$ and $X-i n t \bar{U}$, respectively. Then $\bar{V} \cap W=\varnothing$ and so $\bar{V} \subset X-W \subset$ int $\bar{U}$. In view of Lemma 1.1, it follows that int $\bar{U}$ is $\theta$-open.

To prove the converse, let $F$ be a regularly closed set in $X$ and let $x$ be a point in $X$ outside $F$. Then $x \in X-F$. Since $X-F$ is regularly open, $X-F=\operatorname{int} \overline{X-F}$, which is $\theta$-open. So by Lemma 1.1, there exists an open set $U$ containing $x$ such that $\bar{U} \subset X-F$. Thus $U$ and $X-\bar{U}$ are disjoint open sets containing $x$ and $F$, respectively. Consequently, $X$ is almost regular.

Theorem 2.6. An almost regular $\theta$-compact space is nearly compact. 
Proof. Let $\mathcal{U}$ be an open covering of $X$. By Theorem 2.5, for each $U \in \mathcal{U}$, int $\bar{U}$ is a $\theta$-open set containing $U$ and hence the collection $\{$ int $\bar{U}: U \in \mathcal{U}\}$ is a $\theta$-open covering of $X$. Since $X$ is $\theta$-compact, there exist, a finite subcollection $\left\{U_{1}, \ldots, U_{n}\right\}$ of $\mathcal{U}$ such that $\bigcup_{i=1}^{n} i n t \overline{U_{i}}=X$, and so $X$ is a nearly compact space.

Corollary 2.7. ([21, Theorem 2.3]). An almost regular almost compact space is nearly compact.

Theorem 2.8. An almost regular $\theta$-compact space is mildly normal $(=\kappa$ normal).

Proof. Let $X$ be an almost regular $\theta$-compact space. Let $A$ and $B$ be any two disjoint regularly closed subsets of $X$. Since $X$ is almost regular, for each $b \in B$, there exist disjoint open sets $U_{b}$ and $V_{b}$ containing $A$ and $b$, respectively. Then $U_{b} \cap \overline{V_{b}}=\varnothing$. So by Theorem 2.5, int $\overline{V_{b}}$ is a $\theta$-open set containing $b$ which is disjoint from $U_{b}$. Thus the collection $\left\{\right.$ int $\left.\overline{V_{b}}: b \in B\right\}$ consists of $\theta$-open sets and covers $B$. Let $W=\bigcup_{b \in B} i n t \overline{V_{b}}$. Then $B \subset W$. Let $C=X-W$. Since $B$ is a regularly closed set disjoint from $C$, by almost regularity of $X$, for each $c \in C$, there exist disjoint open sets $X_{c}$ and $W_{c}$ containing $B$ and $c$, respectively. Again, in view of Theorem 2.5, $i n t \overline{W_{c}}$ is a $\theta$-open set which is disjoint from $B$. Hence the collection $\mathcal{C}=\left\{\right.$ int $\left.\overline{V_{b}}: b \in B\right\} \cup\left\{\right.$ int $\overline{W_{c}}$ : $c \in C\}$ is a covering of $X$ by $\theta$-open sets. Since $X$ is $\theta$-compact, there exists a finite subcollection $\mathcal{U}$ of $\mathcal{C}$ which covers $X$. Let $\mathcal{G}$ denote the members of $\mathcal{U}$ which intersect $B$. Since for each $c \in \mathcal{C}$, int $\overline{W_{c}} \cap B=\varnothing$, each member of $\mathcal{G}$ is of the form int $\overline{V_{b}}$ for some $b \in B$. Suppose $\mathcal{G}=\left\{i n t \overline{V_{b_{i}}}: i=1, \ldots n\right\}$. Let $U=\bigcap_{i=1}^{n} U_{b_{i}}$ and $V=\bigcup_{i=1}^{n} i n t \overline{V_{b_{i}}}$. Then $U$ and $V$ are disjoint open sets containing $A$ and $B$, respectively. Hence $X$ is a mildly normal space.

Corollary 2.9. An almost regular almost compact space is mildly normal ( $\kappa$ normal).

With the additional hypothesis of Hausdorffness Theorem 2.8 is strengthened as follows.

Theorem 2.10. A Hausdorff $\theta$-compact space is almost normal if and only if it is almost regular.

Proof. Since a $\mathrm{T}_{1}$-almost normal space is almost regular, necessity is obvious. To prove the sufficiency, let $X$ be a Hausdorff almost regular, $\theta$-compact space. By Theorem 2.6, $X$ is nearly compact and hence almost compact. Since an almost regular Hausdorff space is Urysohn [19] and since an almost compact Urysohn space is almost normal [20], $X$ is almost normal.

We may recall that a space $X$ is an $\mathrm{R}_{0}$-space [5] if every open set in $X$ is the union of closed sets. In [3], $\mathrm{R}_{0}$-spaces are referred to as $\mathrm{S}_{1}$-spaces. It is shown 
in [3, p. 196] that in an $\mathrm{R}_{0}$-space the closure of every singleton is compact. For $\theta$-closure we have the following.

Theorem 2.11. In a topological space $X$ the $\theta$-closure of every singleton is a $\theta$-set.

Proof. Let $\mathcal{U}$ be a covering of $c l_{\theta}\{x\}$ by $\theta$-open sets in $X$. Let $x \in U \in \mathcal{U}$. By Lemma 1.1, there exists an open set $V$ containing $x$ such that $\bar{V} \subset U$. In view of [28, Lemma 2], $c l_{\theta}\{x\} \subset c l_{\theta} V=\bar{V} \subset U$. Hence $c l_{\theta}\{x\}$ is a $\theta$-set.

Remark 2.12. Theorem 2.11 cannot be strengthened to read " $\theta$-compact" instead of " $\theta$-set". For example, let $X=\{a\} \cup B \cup C$ with pairwise disjoint members and infinite sets $B$ and $C$. Let the topology $\tau$ be defined by the base $\{\{c\},(\{a\} \cup U),(\{b\} \cup U): b \in B, c \in C, U \subset C$ is cofinite in $C\}$. Clearly, $c l_{\theta}(\{a\})=\{a\} \cup B$ which is discrete and infinite in the subspace topology.

\section{Subspaces}

The following formulation of the notion of a $\theta$-embedded set is useful in studying subspaces of $\theta$-compact spaces.

Definition 3.1. A subset $Y$ of a topological space $X$ is said to be $\theta$-embedded in $X$ if every $\theta$-closed set in the subspace topology of $Y$ is the intersection of $Y$ with a $\theta$-closed set in $X$.

Remark 3.2. Let $X$ be the same space as in Remark 2.2. The set $A$ therein is a $\theta$-closed subset of $X$ which is not $\theta$-embedded in $X$. The same example also shows that a $\theta$-closed subset of a $\theta$-compact space need not be $\theta$-compact. However, the following is true.

Theorem 3.3. A $\theta$-closed, $\theta$-embedded subset of a $\theta$-compact space is $\theta$-compact.

Proof. Let $A$ be a $\theta$-closed, $\theta$-embedded subset of a $\theta$-compact space $X$. Let $\mathcal{U}$ be a covering of $A$ by sets $\theta$-open in $A$. Since $A$ is $\theta$-embedded in $X$, for each $U_{\alpha} \in \mathcal{U}$ there exists a $\theta$-open set $V_{\alpha}$ in $X$ such that $U_{\alpha}=A \cap V_{\alpha}$. Then the collection $\mathcal{V}=\left\{V_{\alpha}: U_{\alpha} \in \mathcal{U}\right\} \cup\{X-A\}$ is a $\theta$-open covering of $X$. By $\theta$-compactness of $X$, there is a finite subcollection $\left\{V_{\alpha_{1}}, \ldots, V_{\alpha_{n}}\right\}$ of $\mathcal{V}$ which covers $X$. Then the collection $\left\{V_{\alpha_{1}} \cap A, \ldots, V_{\alpha_{n}} \cap A\right\}$ is a finite subcollection of $\mathcal{U}$ which covers $A$ and so $A$ is $\theta$-compact.

Corollary 3.4. Every clopen subset of a $\theta$-compact space is $\theta$-compact.

A topological space $X$ is said to be $\theta$-Hausdorff [23] if any two distinct points in $X$ are contained in disjoint $\theta$-open sets. A space $X$ is called a locally $\theta$-space [4] if each $x \in X$ has a neighbourhood which is a $\theta$-set.

To conclude this section we quote the following theorem from [4].

Theorem 3.5. A non $\theta$-compact, $\theta$-Hausdorff, locally $\theta$-space $X$ has a one point Hausdorff $\theta$-compactification, i.e., it is a dense open subspace of a $\theta$ compact Hausdorff space $X^{*}$ such that $X^{*}-X$ is a singleton. 
4. DiRECT AND INVERSE PRESERVATION UNDER MAPPINGS AND PRODUCTS.

Definition 4.1. A function $f: X \rightarrow Y$ is said to be

(i) $\theta$-continuous [7] if for each $x \in X$ and each open set $U$ containing $f(x)$ there exists an open set $V$ containing $x$ such that $f(\bar{V}) \subset \bar{U}$, and

(ii) strongly $\theta$-continuous [16] if for each $x \in X$ and each open set $U$ containing $f(x)$ there exists an open set $V$ containing $x$ such that $f(\bar{V}) \subset U$.

We say that a function $f: X \rightarrow Y$ is a $\theta$-map if for every $\theta$-open set $U$ in $Y, f^{-1}(U)$ is $\theta$-open in $X$.

Theorem 4.2. Every $\theta$-continuous function is a $\theta$-map.

Proof. Let $f: X \rightarrow Y$ be a $\theta$-continuous function and let $U$ be a $\theta$-open set in $Y$. Let $x \in f^{-1}(U)$. Then, $f(x) \in U$. Since $U$ is $\theta$-open, by Lemma 1.1 , there exists an open set $V$ in $Y$ such that $f(x) \in V \subset \bar{V} \subset U$. By $\theta$-continuity of $f$, there exists an open set $W$ in $X$ containing $x$ such that $f(\bar{W}) \subset \bar{V} \subset U$. Thus $x \in W \subset \bar{W} \subset f^{-1}(U)$. So in view of Lemma 1.1, $f^{-1}(U)$ is $\theta$-open and hence $f$ is a $\theta$-map.

Remark 4.3. The converse of Theorem 4.2 is not true. For let $X=Y$ be the set of positive integers. Let $X$ be endowed with the cofinite topology and let $Y$ be equipped with the topology as defined in Example 2.3. Then the identity mapping of $X$ onto $Y$ is a $\theta$-map which is not $\theta$-continuous.

Theorem 4.4. Let $f: X \rightarrow Y$ be a $\theta$-map from a $\theta$-compact space $X$ onto $Y$. Then $Y$ is $\theta$-compact.

Proof. Let $\mathcal{V}$ be a $\theta$-open covering of $Y$. Then since $f$ is a $\theta$-map, the collection $\mathcal{U}=\left\{f^{-1}(U): U \in \mathcal{V}\right\}$ is a $\theta$-open covering of $X$. Since $X$ is $\theta$-compact, there exists a finite subcollection $\left\{f^{-1}\left(U_{i}\right): i=1, \ldots, n\right\}$ of $\mathcal{U}$ which covers $X$. Now since $f$ is onto, $\left\{U_{i}: i=1, \ldots, n\right\}$ is a finite subcollection of $\mathcal{V}$ which covers $Y$. Hence $Y$ is a $\theta$-compact space.

Corollary 4.5. Every $\theta$-continuous image of a $\theta$-compact space is $\theta$-compact.

Theorem 4.6. A strongly $\theta$-continuous image of a $\theta$-compact space is compact.

Proof. Suppose $X$ is a $\theta$-compact space and let $f: X \rightarrow Y$ be a strongly $\theta$ continuous surjection. Let $\mathcal{V}$ be an open covering of $Y$. Let $V \in \mathcal{V}$. We show that $f^{-1}(V)$ is $\theta$-open. If $x \in f^{-1}(V)$, then $f(x) \in V$. Since $f$ is strongly $\theta$-continuous, there exists an open set $U$ containing $x$ such that $f(\bar{U}) \subset V$. Then $x \in U \subset \bar{U} \subset f^{-1}(V)$ and so in view of Lemma 1.1, $f^{-1}(U)$ is $\theta$-open. Thus the collection $\mathcal{U}=\left\{f^{-1}(V): V \in \mathcal{V}\right\}$ is a $\theta$-open covering of $X$ and so there is a finite subcollection $\left\{f^{-1}\left(V_{i}\right): i=1,2, \ldots n\right\}$ of $\mathcal{U}$ which covers $X$. Hence $\left\{V_{i}: i=1,2, \ldots, n\right\}$ is a finite subcollection of $\mathcal{V}$ which covers $Y$ and so $Y$ is compact. 
We say that a function $f: X \rightarrow Y$ is said to be $\theta$-closed if each $\theta$-closed set $F$ in $X, f(F)$ is $\theta$-closed.

The following characterization of $\theta$-closed functions will be used in the sequel and seems to be of interest in itself.

Theorem 4.7. A function $f: X \rightarrow Y$ is $\theta$-closed if and only if for each set $B \subset Y$ and for each $\theta$-open set $U$ containing $f^{-1}(B)$, there is a $\theta$-open set $V$ containing $B$ such that $f^{-1}(V) \subset U$.

Proof. Necessity. Since $U$ is $\theta$-open, $X-U$ is $\theta$-closed and so $f(X-U)$ is $\theta$-closed in $Y$. Now, $V=Y-f(X-U)$ is $\theta$-open, $B \subset V$ and

$f^{-1}(V)=f^{-1}(Y-f(X-U))=X-f^{-1}(f(X-U)) \subset X-(X-U)=U$.

To prove sufficiency, let $A$ be a $\theta$-closed set in $X$. To prove that $f(A)$ is $\theta$-closed, we shall show that $Y-f(A)$ is $\theta$-open. Let $y \in Y-f(A)$. Then $f^{-1}(y) \cap f^{-1}(f(A))=\phi$ and so $f^{-1}(y) \subset X-f^{-1}(f(A)) \subset X-A$. By hypothesis there exists a $\theta$-open set $V$ containing $y$ such that $f^{-1}(V) \subset X-A$. So $A \subset X-f^{-1}(V)$ and hence $f(A) \subset f\left(X-f^{-1}(V)\right)=Y-V$. Thus $V \subset Y-f(A)$ and so the set $Y-f(A)$ being the union of $\theta$-open sets is $\theta$-open.

Theorem 4.8. Let $f: X \rightarrow Y$ be a $\theta$-closed surjection such that for each $y \in Y, f^{-1}(y)$ is a $\theta$-compact subset of $X$. If $Y$ is $\theta$-compact, then so is $X$.

Proof. Let $\mathcal{U}=\left\{U_{\alpha}: \alpha \in \Lambda\right\}$ be a $\theta$-open covering of $X$. Since for each $y \in Y$, $f^{-1}(y)$ is a $\theta$-compact subset of $X$, we can choose a finite subset $\Lambda_{y}$ of $\Lambda$ such that $\left\{U_{\beta}: \beta \in \Lambda_{y}\right\}$ is a covering of $f^{-1}(y)$. Now, by Theorem 4.7, there exists a $\theta$-open set $V_{y}$ containing $y$ such that $f^{-1}\left(V_{y}\right) \subset \cup\left\{U_{\beta}: \beta \in \Lambda_{y}\right\}$. The collection $\mathcal{V}=\left\{V_{y}: y \in Y\right\}$ is a $\theta$-open covering of $Y$. In view of $\theta$-compactness of $Y$ there exists a finite subcollection $\left\{V_{y_{1}}, \ldots, V_{y_{n}}\right\}$ of $\mathcal{V}$ which covers $Y$. Then the finite subcollection $\left\{U_{\beta}: \beta \in \Lambda_{y_{i}}, i=1, \ldots, n\right\}$ of $\mathcal{U}$ covers $X$. Hence $X$ is a $\theta$-compact space.

Lemma 4.9. Let $X$ be a compact space. Then the projection map $p_{y}: X \times Y \rightarrow$ $Y$ is a $\theta$-closed surjection for any space $Y$.

Proof. Let $F$ be a $\theta$-closed subset of $X \times Y$. To show that $p_{y}(F)$ is $\theta$-closed, we prove that $Y-p_{y}(F)$ is $\theta$-open. Let $y \in Y-p_{y}(F)$. Then $(X \times\{y\}) \cap F$ $=\varnothing$. Since $(X \times Y)-F$ is $\theta$-open, for each $(x, y)$, there is a basic open set $U(x) \times V_{y}(x)$ containing $(x, y)$ such that $\overline{U(x)} \times \overline{V_{y}(x)}$ is disjoint from $F$. The collection $\left\{U(x) \times V_{y}(x): x \in X\right\}$ is an open covering of the compact set $X \times\{y\}$ and so has a finite subcovering $\left\{U\left(x_{i}\right) \times V_{y}\left(x_{i}\right): i=1, \ldots, n\right\}$. Then $V=\bigcap_{i=1}^{n} V_{y}\left(x_{i}\right)$ is an open set containing $y$ such that $\bar{V} \subset Y-p_{y}(F)$. In view of Lemma 1.1, $Y-p_{y}(F)$ is $\theta$-open and hence $p_{y}$ is a $\theta$-closed surjection.

The following corollary concerning $\theta$-open sets in the product space $X \times Y$ is analogous to its counterpart for open sets which is widely used in applications. 
Corollary 4.10. Let $Y$ be a compact space and let $A \subset X$ be arbitrary. Let $U$ be a $\theta$-open set in $X \times Y$ containing $A \times Y$. Then there is a $\theta$-open set $V$ containing $A$ such that $V \times Y \subset U$.

Proof. By Lemma 4.9, the projection map $p_{x}: X \times Y \rightarrow X$ is a $\theta$-closed function. Now, $p_{x}^{-1}(A)=A \times Y \subset U$. So an application of Theorem 4.7, yields the desired $\theta$-open set $V$ containing $A$ such that $p_{x}^{-1}(V)=V \times Y \subset U$.

Theorem 4.11. Let $X$ and $Y$ be $\theta$-compact spaces such that $X$ is compact. Then $X \times Y$ is a $\theta$-compact space.

Proof. By Lemma 4.9, the projection map $p_{y}: X \times Y \rightarrow Y$ is a $\theta$-closed surjection. Again, for each $y \in Y$, the fiber $p_{y}^{-1}(y)$ is compact and hence $\theta$-compact. So the result is immediate in view of Theorem 4.8 .

\section{Characterizations and a factorization of CompaCtness.}

In this section we obtain characterizations of $\theta$-compactness analogous to that of compactness.

In general $\theta$-closure operator is not a Kuratowski closure operator, since $\theta$-closure of a set may not be $\theta$-closed (see [10]). However, the following modification yields a Kuratowski closure operator.

Definition 5.1. Let $X$ be a topological space and let $A \subset X$. A point $x \in X$ is called a $u \theta$-limit point of $A$ if every $\theta$-open set $U$ containing $x$ intersects $A$. The set of all $u \theta$-limit points of $A$ is either denoted by $A_{u \theta}$ or by $c_{u \theta} A$. The set $A$ is called $u \theta$-closed if $A=A_{u \theta}$.

Lemma 5.2. The operator $A \rightarrow A_{u \theta}$ is a Kuratowski closure operator.

Proof. Clearly $A \subset A_{u \theta}$, for every subset $A$ of $X$. Clearly, $\left(A_{u \theta} \cup B_{u \theta}\right) \subset(A \cup$ $B)_{u \theta}$. To prove the reverse inequality $(A \cup B)_{u \theta} \subset A_{u \theta} \cup B_{u \theta}$, let $x \in(A \cup B)_{u \theta}$. Suppose $x \notin A_{u \theta} \cup B_{u \theta}$. Then $x \notin A_{u \theta}$ and $x \notin B_{u \theta}$. So there exist $\theta$-open sets $U$ and $V$ containing $x$ such that $(U \cap A)=\varnothing=(V \cap B)$. Then $U \cap V$ is a $\theta$-open set containing $x$ such that $(U \cap V) \cap(A \cup B)=\varnothing$, which contradicts the fact that $x \in(A \cup B)_{u \theta}$. Consequently, $A_{u \theta} \cup B_{u \theta}=(A \cup B)_{u \theta}$. To prove $\left(A_{u \theta}\right)_{u \theta} \subset A_{u \theta}$, let $x \in\left(A_{u \theta}\right)_{u \theta}$. Then for every $\theta$-open set $U$ containing $x$, $U \cap A_{u \theta} \neq \varnothing$. Choose $y \in U \cap A_{u \theta}$. Since $U$ is a $\theta$-open set containing $y$, $U \cap A \neq \varnothing$ and so $x \in A_{u \theta}$. Hence $\left(A_{u \theta}\right)_{u \theta}=A_{u \theta}$. This completes the proof that the correspondence $A \rightarrow A_{u \theta}$ is a Kuratowski closure operator.

Remark 5.3. For any set $A \subset X, A \subset \bar{A} \subset A_{\theta} \subset A_{u \theta}$ and $A_{u \theta}$ is the smallest $\theta$-closed set containing $A$.

Definition 5.4. Let $\Im$ be a filter on $X$. A point $x \in X$ is said to be $u \theta$-cluster point of $\Im$ if every $\theta$-open set containing $x$ intersects every member of the filter $\Im$. The filter $\Im$ is said to $u \theta$-converges to $x$ if every $\theta$-open set containing $x$ belongs to $\Im$. In symbols $\Im \stackrel{u \theta}{\longrightarrow} x$. 
Definition 5.5. Let $\left\{x_{\alpha}\right\}_{\alpha \in \Lambda}$ be a net in $X$. A point $x$ is said to be a $\boldsymbol{u} \theta$ cluster point of $\left\{x_{\alpha}\right\}$ if for every $\theta$-open set $U$ containing $x$ and for each $\alpha_{0} \in \Lambda$ there is an $\alpha \in \Lambda$ such that $\alpha>\alpha_{0}$ and $x_{\alpha} \in U$. The net $\left\{x_{\alpha}\right\}$ is said to be $u \theta$-converges to $x$ if for each $\theta$-open set containing $x$, there is some $\lambda_{0} \in \Lambda$ such that $x_{\lambda} \in U$ for all $\lambda \geq \lambda_{0}$.

Proofs of following four lemmas are not so very different from those in the classical case of convergence and cluster points of nets and filters and hence omitted.

Lemma 5.6. A point $x$ is a $u \theta$-cluster point of a filter $\Im$ if and only if there is a filter $\Re$ finer than $\Im$ and $\Re \stackrel{u \theta}{\longrightarrow} x$.

Lemma 5.7. An ultranet $u \theta$-converges to each of its $u \theta$-cluster points.

Lemma 5.8. A point $x \in X$ is a $u \theta$-cluster point of a net $\left\{x_{\alpha}\right\}$ if and only if $x$ is a $u \theta$-cluster point of the filter generated by the net $\left\{x_{\alpha}\right\}$.

Lemma 5.9. A filter $\Im u \theta$-converges to $x$ if and only if the net based on $\Im$ $u \theta$-converges to $x$.

Theorem 5.10. For a topological space $X$, the following statements are equivalent.

(a) $X$ is $\theta$-compact.

(b) Every family of $\theta$-closed sets with finite intersection property has a nonempty intersection.

(c) Each filter on $X$ has a $u \theta$-cluster point in $X$.

(d) Each net in $X$ has a $u \theta$-cluster point in $X$.

(e) Each ultranet in $X$ is $u \theta$-convergent.

(f) Each ultrafilter on $X$ is $u \theta$-convergent.

Proof. The assertion of (a) $\Rightarrow(\mathrm{b})$ is easy.

To prove $(\mathrm{b}) \Rightarrow(\mathrm{c})$, let $\Im$ be a filter on $X$. Suppose that $\Im$ has no $u \theta$-cluster point in $X$. Then for each $x \in X$, there exists a $\theta$-open set $U_{x}$ containing $x$ such that $U_{x} \cap F_{x}=\varnothing$ for some $F_{x}$ in $\Im$ and so $x \notin c l_{u \theta} F_{x}$. Thus the collection $\left\{c l_{u \theta} F: F \in \Im\right\}$ is a family of $\theta$-closed sets with finite intersection property which has empty intersection.

The implication $(\mathrm{c}) \Rightarrow(\mathrm{d})$ is immediate in view of Lemma 5.8 and $(\mathrm{d}) \Rightarrow$ (e) follows from Lemma 5.7. Similarly, the assertion (e) $\Rightarrow$ (f) is clear from Lemma 5.9.

To prove (f) $\Rightarrow(\mathrm{a})$, suppose that each ultrafilter on $X$ is $u \theta$-convergent. Let $\mathcal{V}$ be a covering of $X$ by $\theta$-open sets. If $\mathcal{V}$ has no finite subcovering of $X$, then the collection $\{X-V: V \in \mathcal{V}\}$ is a filter base and so is contained in an ultrafilter $\mathcal{G}$. By hypothesis the ultrafilter $\mathcal{G}$ is $u \theta$-convergent to $x$, say. Let $V \in \mathcal{V}$ be a $\theta$-open set containing $x$. Since $\mathcal{G} \stackrel{u \theta}{\longrightarrow} x, V \in \mathcal{G}$. But $X-V \in \mathcal{G}$. This contradiction proves that $\mathcal{V}$ has a finite subcovering.

A space $X$ is said to be point paracompact [2] if for every open covering $\mathcal{U}$ of $X$ and each $x \in X$ there is an open refinement $\mathcal{V}$ of $\mathcal{U}$ such that $\mathcal{V}$ is 
locally finite at $x$. We say that a space $X$ is $\theta$-point paracompact if for each open covering $\mathcal{U}$ of $X$ and each $x \in X$ there is an open refinement $\mathcal{V}$ of $\mathcal{U}$ and a $\theta$-open set $U$ containing $x$ which intersects only finitely many members of $\mathcal{V}$.

It is clear from the definitions that every compact space is $\theta$-point paracompact and that a $\theta$-point paracompact space is point paracompact. The space $X$ in Example 2.3 is a point paracompact space which is not $\theta$-point paracompact.

Theorem 5.11. A topological space $X$ is $\theta$-point paracompact if and only if every open covering of $X$ has, for each $x \in X$, a finite subset whose union contains the closure of some $\theta$-open set containing $x$.

Proof. Suppose $X$ is $\theta$-point paracompact space and let $\mathcal{U}=\left\{U_{\alpha}: \alpha \in \Lambda\right\}$ be an open covering of $X$. Suppose $x \in X$. Then there exists an open refinement $\left\{V_{\lambda}: \lambda \in \Lambda_{x}\right\}$ of $\mathcal{U}$ and a $\theta$-open set $N(x)$ containing $x$ such that $N(x) \cap V_{\lambda} \neq \varnothing$ for only finitely many $\lambda \in \Lambda_{x}$. It follows that there exists a finite subset $\left\{U_{\alpha(k)}: k=1, \ldots, n\right\}$ of $\mathcal{U}$ such that $\overline{N(x)} \subset \bigcup_{k=1}^{n} U_{\alpha(k)}$.

Conversely, let $\left\{U_{\alpha}: \alpha \in \Lambda\right\}$ be an open covering of $X$ and let $x \in X$. So by hypothesis there exists a $\theta$-open set $N(x)$ containing $x$ and a finite subset $\left\{U_{\alpha(i)}: i=1, \ldots, n\right\}$ of $\mathcal{U}$ such that $\overline{N(x)} \subset \bigcup_{i=1}^{n} U_{\alpha(i)}$. Let

$\mathcal{V}=\left\{\left(X-\overline{N(x)} \cap U_{\alpha}: \alpha \in \Lambda\right\} \cup\left\{U_{\alpha(i)}: i=1, \ldots, n\right\}\right.$.

Clearly, $\mathcal{V}$ is a refinement of $\mathcal{U}$ with $N(x)$ intersecting only finitely many members of $\mathcal{V}$. So $X$ is $\theta$-point paracompact.

Corollary 5.12. Every regular space is $\theta$-point paracompact.

Corollary 5.13 ([2]). Every regular space is point paracompact.

Theorem 5.14. If $X$ is a $\theta$-point paracompact space then every filter with a $u \theta$-cluster point has a cluster point.

Proof. Let $X$ be $\theta$-point paracompact space and let $\Im$ be a filter with no cluster point. Let $x \in X$, then $\mathcal{U}=\{X-\bar{F}: F \in \Im\}$ is a directed open covering of $X$. By the hypothesis, there exists an open refinement $\mathcal{V}$ of $\mathcal{U}$ which is $\theta$-locally finite at $x$. So there exists a $\theta$-open set $V$ containing $x$ which intersects only finitely many members of $\mathcal{V}$. Let $W=\cup\{U: U \in \mathcal{V}, U \cap V=\varnothing\}$. We have $V \cap W=\varnothing$. Now $X-W \subseteq \cup\{U: U \in \mathcal{V} ; U \cap V \neq \varnothing\}$. Since $\mathcal{V}$ is a refinement of $\mathcal{U}$ there exist finitely many $O_{i}$ 's in $\mathcal{U}$ such that $\cup\{U \in \mathcal{V}$; $U \cap V \neq \varnothing\} \subseteq \bigcup_{i=1}^{n}\left\{O_{i}: O_{i} \in \mathcal{U}\right\}$. As $\mathcal{U}$ is a directed cover, there is some $F$ in $\Im$ such that $\bigcup_{i=1}^{n}\left\{O_{i}: O_{i} \in \mathcal{U}\right\} \subseteq X-\bar{F}$. Thus $X-W \subseteq X-\bar{F}$. i.e., $\bar{F} \subseteq W$. Hence $V$ is a $\theta$-open set containing $x$ and $V \cap F=\varnothing$. Therefore $x$ cannot be a $u \theta$-limit point of $\Im$.

A combination of Theorems 5.10(c) and 5.14, yields the following factorization of compactness. 
Theorem 5.15. A topological space $X$ is compact if and only if it is both a $\theta$-compact space and a $\theta$-point paracompact space.

Corollary 5.16. A regular $\theta$-compact space is compact.

Corollary $\mathbf{5 . 1 7}$ ([3, 27]). A regular almost compact space is compact.

\section{REFERENCES}

[1] A. J. D'Aristotle, Quasicompactness and functionally Hausdorff spaces, J. Austral. Math. Soc. 15 (1973), 319-324.

[2] J. M. Boyte, Point (countable) paracompactness, J. Austral. Math. Soc.,15 (1973), 138144.

[3] Á. Császár, General Topology, Adam Higler Ltd, Bristol, 1978.

[4] A. K. Das, A note on $\theta$-Hausdorff spaces, Bull. Cal. Math. Soc. 97(1) (2005), 15-20.

[5] A. S. Davis, Indexed systems of neighbourhoods for general topological spaces, Amer. Math. Monthly 68 (1961), 886-893.

[6] R. F. Dickman and J. R. Porter, $\theta$-perfect and $\theta$-absolutely closed functions, Illinois J. Math. 21 (1977), 42-60.

[7] S. Fomin, Extensions of topological spaces, Ann. Math. 44 (1943), 471-480.

[8] Z. Frolik, Generalizations of compact and Lindelöf spaces, Czechoslovak Math J. 13 (84) (1959), 172-217 (Russian) MR 213821.

[9] E. Hewitt, On two problems of Urysohn, Ann. Math. (2) 47 (1946), 503-509.

[10] J. E. Joseph, $\theta$-closure and $\theta$-subclosed graphs, Math. Chron. 8 (1979), 99-117.

[11] J. K. Kohli and A. K. Das, New normality axioms and decompositions of normality, Glasnik Mat. 37(57)(2002), 163-173.

[12] J. K. Kohli and A. K. Das, On functionally $\theta$-normal spaces, Applied General Topology 6(1) (2005), 1-14.

[13] J. K. Kohli, A. K. Das and R. Kumar, Weakly functionally $\theta$-normal spaces, $\theta$-shrinking of covers and partition of unity, Note di Matematica, 19(2)(1999), 293-297.

[14] C. Kuratowski, Topologie I, Hafner, New York, 1958.

[15] C. T. Liu, Absolutely closed spaces, Trans. Amer. Math. Soc. 130 (1968), 68-104.

[16] T. Noiri, $\delta$-continuous functions, J. Korean Math. Soc. 16 (1980), 161-166.

[17] J. R. Porter and J. Thomas, On H-closed spaces and minimal Hausdorff spaces, Trans. Amer. Math. Soc. 138 (1969), 159-170.

[18] C. T. Scarborough and A. H. Stone, Products of nearly compact spaces, Trans. Amer. Math. Soc. 124 (1966), 131-147.

[19] M. K. Singal and S. P. Arya, On almost regular spaces, Glasnik Mat. 4 (24) (1969), 89-99.

[20] M. K. Singal and S. P. Arya, On almost normal and almost completely regular spaces, Glasnik Mat. 5(25)(1970), 141-152.

[21] M. K. Singal and A. Mathur, On nearly compact spaces, Boll. U.M.I. 2(4) (1969), 702710.

[22] M. K. Singal and A. R. Singal, Mildly normal spaces, Kyungpook Math. J. 13(1) (1973), $27-31$.

[23] S. Sinharoy and B. Bandopadhyay, On $\theta$-completely regular and locally $\theta-H$ closed spaces, Bull. Cal. Math. Soc. 87 (1995), 19-28.

[24] E. V. Stchepin, Real valued functions and spaces close to normal, Sib. J. Math. 13:5 (1972), 1182-1196.

[25] R. M. Stephenson, Jr., Spaces for which the Stone-Weierstrass theorem holds, Trans. Amer. Math. Soc. 133 (1968), 537-546.

[26] R. M. Stephenson, Jr., Product spaces for which Stone-Weierstrass theorem holds, Proc. Amer. Math. Soc. 21 (1969), 284-288.

[27] W. J. Thron, Topological Structures, Holt, Rinehart and Winston, New York (1966). 
[28] N. V. Veličko, H-closed topological spaces, Amer. Math. Soc. Transl. 2, 78 (1968), 103-118.

ReCEIVEd May 2005

AcCePted November 2005

J. K. KoHLI

Department of Mathematics, Hindu College, University of Delhi, Delhi-110007, India

A. K. DAS (ak_das@lycos.com)

Department of Mathematics, Bhim Rao Ambedkar College, University Of Delhi, Yamuna Vihar, Delhi-110094, India 